\title{
Remarks on superlinear boundary value problems
}

\section{Svatopluk Fuxík}

\begin{abstract}
Necessary and sufficient conditions for the weak solvability of the Dirichlet problem for nonlinear differential equations of the second order are proved. The differential operators considered are in the form of a sum of a linear noninvertible operator, with the null-space generated by a positive function, and a monotone nonlinear perturbation, the growth of which is more than linear.
\end{abstract}

\section{Introduction}

Let $\Omega$ be a bounded domain in $R^{N}(N \geq 1)$ with the boundary $\partial \Omega$ which is locally lipschitzian if $N \geq 2$. We define the Sobolev space $W^{1,2}(\Omega)$ as the subspace of $L_{2}(\Omega)$ consisting of all real-valued functions $u$ for which $\frac{\partial u}{\partial x_{i}} \in L_{2}(\Omega), i=1, \ldots, N\left(\frac{\partial}{\partial x_{i}}\right.$ means the derivative in the sense of distributions). $W^{7,2}(\Omega)$ is a Hilbert space with the inner product

$$
\langle u, v\rangle=\sum_{i=1}^{N} \int_{\Omega} \frac{\partial u(x)}{\partial x_{i}} \frac{\partial v(x)}{\partial x_{i}} d x+\int_{\Omega} u(x) v(x) d x
$$

and the norm $\|u\|=(u, u)^{\frac{3}{2}}$. Further, denoting by $D(\Omega)$ the set of all infinitely differentiable functions on $\Omega$ with compact supports in $\Omega$, we define $W_{0}^{1,2}(\Omega)$ as the closure of $D(\Omega)$ in $W^{1,2}(\Omega)$.

Received 20 October 1976. 
Let $\lambda \in R^{l}$. Let $g: R^{l} \rightarrow R^{l}$ be a continuous function satisfying in the case of $N \geq 2$ certain growth conditions and let $f \in L_{p}(\Omega)$ (for precise formulation see the theorem). The function $u \in W_{0}^{l, 2}(\Omega)$ is said to be a weak solution of the Dirichlet problem

$$
\left\{\begin{array}{l}
-\Delta u(x)-\lambda u(x)+g(u(x))=f(x), x \in \Omega, \\
u(x)=0, x \in \partial \Omega,
\end{array}\right.
$$

if the integral identity

$$
\sum_{i=1}^{N} \int_{\Omega} \frac{\partial u(x)}{\partial x_{i}} \frac{\partial \varphi(x)}{\partial x_{i}} d x-\lambda \int_{\Omega} u(x) \varphi(x) d x+\int_{\Omega} g(u(x)) \varphi(x) d x=\int_{\Omega} f(x) \varphi(x) d x
$$

holds for arbitrary $\varphi \in W_{0}^{I, 2}(\Omega)$. It is well-known that the set of all parameters $\lambda$ for which the linear Dirichlet problem

$$
\left\{\begin{array}{l}
-\Delta u-\lambda u=0 \text { in } \Omega, \\
u=0 \text { on } \partial \Omega
\end{array}\right.
$$

has a nontrivial weak solution forms a sequence $\left\{\lambda_{m}\right\}, 0<\lambda_{1}<\lambda_{2}<\ldots$, such that $\lim \lambda_{m}=+\infty$. Furthermore, the set $\operatorname{ker}\left[-\Delta-\lambda_{1}\right]$ of all weak solutions of (2) with $\lambda=\lambda_{1}$ is a one-dimensional subspace of $w_{0}^{l, 2}(\Omega)$ generated by the function $w_{0} \in W_{0}^{l}, 2(\Omega)$, which is positive in $\Omega$.

We are concerned with the weak solvability of the nonlinear Dirichlet problem

$$
\left\{\begin{array}{l}
-\Delta u(x)-\lambda_{1} u(x)+g(u(x))=f(x), x \in \Omega, \\
u(x)=0, x \in \partial \Omega .
\end{array}\right.
$$

Our main result is the following theorem.

THEOREM. Let $g: R^{1} \rightarrow R^{1}$ be a continuous nondecreasing function, increasing on some interval $(-\varepsilon, \varepsilon)$. Moreover, if $N \geq 2$ we suppose that $g$ is bounded from below and satisfies the growth condition 


$$
|g(\xi)| \leq a+b|\xi|^{\tau}, \quad \xi \in R^{\perp},
$$

where

$$
\tau=\left\{\begin{array}{l}
\text { an arbitrary positive number if } N=2, \\
\frac{N+2}{N-2} \quad \text { if } N \geq 3 .
\end{array}\right.
$$

Denote

$$
g(+\infty)=\lim _{\xi \rightarrow+\infty} g(\xi), g(-\infty)=\lim _{\xi \rightarrow-\infty} g(\xi) .
$$

Let $f \in L_{p}(\Omega)$, where

$$
r=\left\{\begin{array}{l}
1, \text { if } N=1, \\
2, \text { if } N \geq 2 .
\end{array}\right.
$$

(i) If the boundary value problem (3) has at least one weak solution, we have

$$
g(+\infty) \int_{\Omega} w_{0}(x) d x \geq \int_{\Omega} f(x) w_{0}(x) d x \geq g(-\infty) \int_{\Omega} w_{0}(x) d x
$$

(ii) If there holds

$$
g(+\infty) \int_{\Omega} w_{0}(x) d x>\int_{\Omega} f(x) w_{0}(x) d x>g(-\infty) \int_{\Omega} w_{0}(x) d x,
$$

then the boundary value problem (3) has at least one weak solution.

(iii) If we have

$$
g(-\infty)<g(\xi)<g(+\infty), \quad \xi \in R^{l},
$$

then the inequalities (6) are necessary and sufficient conditions for the weak solvability of the boundary value problem (3).

Note that if $\lambda<\lambda_{1}$ and if $g$ satisfies the assumptions of the theorem, then the boundary value problem (1) has, for arbitrary $f \in L_{r}(\Omega)$, at least one solution. This assertion follows immediately by applying the theorem about the surjectivity of monotone, continuous, and coercive operators (see, for example, [2]). If $\lambda \geq \lambda_{1}$ then there are 
known results on weak solvability of (I), provided the function $g$ satisfies the growth condition (4) with $0 \leq \tau \leq 1$ (see, for example, [1], [3-7], [9]). If, however, the growth of $g$ is superlinear (that is $\tau>I$ ) it seems that the only result known is that by Kazdan and Warner (see [8]) on the classical solvability. The proof used in [8] is based on the method of sub and super solutions. The present paper deals with the weak solutions and the method used is quite different: we solve the bifurcation system; the solution of the one-dimensional equation is obtained from the results of classical analysis, the solution of the infinite-dimensional equation is obtained by applying the theory of monotone operators.

A typical example of the Dirichlet problems considered in the theorem is the two-point boundary value problem

$$
\left\{\begin{array}{l}
-u^{\prime \prime}(x)-\lambda u(x)+e^{u(x)}=f(x), x \in(0, \pi), \\
u(0)=u(\pi)=0 .
\end{array}\right.
$$

For $\lambda<1$ and an arbitrary $f \in L_{1}(0, \pi)$, the boundary value problem (8) has a unique solution, and (using the regularity result) for an arbitrary $f \in C([0, \pi])$ there is a unique classical solution. If $\lambda=1$, the boundary value problem (8) has at least one solution for $f \in L_{1}(0, \pi)$ if and only if

$$
\int_{0}^{\pi} f(x) \sin x d x>0
$$

and the inequality ( 9 ) is also a necessary and sufficient condition for the classical solvability of $(8)$, provided $f \in C([0, \pi])$. The problem of both the weak and the classical solvability of (8) in the case of $\lambda>1$ seems to be open.

Finally, we note that it is possible without trouble to replace the Laplace operator by a second order uniformly elliptic selfadjoint operator.

\section{Proof of the theorem}

If the boundary value problem (3) has a weak solution $u_{0} \in w_{0}^{1}, 2(\Omega)$, then 


$$
\int_{\Omega} f(x) w_{0}(x) d x=\int_{\Omega} g\left(u_{0}(x)\right) w_{0}(x) d x,
$$

and from (10) we obtain immediately (5).

Note that (iii) follows from $(i)$ and $(i i)$.

Thus we have to prove $(i i)$. Let $f \in L_{p}(\Omega)$ satisfy the inequalities (6).

1. It is easy to see that the Dirichlet problem ( 3 ) is weakly solvable if and only if there exists a $t \in R^{I}$ and a

$$
v \in X=\left\{\psi \in W_{0}^{1,2}(\Omega) ;\left\langle\psi, w_{0}\right\rangle=0\right\},
$$

such that

$$
\int_{\Omega} g\left(w_{0}(x)+v(x)\right) w_{0}(x) d x=\int_{\Omega} f(x) w_{0}(x) d x
$$

and that the integral identity

$$
\begin{aligned}
\sum_{i=1}^{N} \int_{\Omega} \frac{\partial v(x)}{\partial x_{i}} \frac{\partial \psi(x)}{\partial x_{i}} d x-\lambda_{1} & \int_{\Omega} v(x) \psi(x) d x+ \\
& +\int_{\Omega} g\left(\omega_{0}(x)+v(x)\right) \cdot \psi(x) d x=\int_{\Omega} f(x) \psi(x) d x
\end{aligned}
$$

holds for arbitrary $\psi \in X$.

2. Let $v \in X$ be arbitrary but fixed. Then there exists a uniquely determined $t(v) \in R^{l}$ such that (11) holds for $t=t(v)$ (this follows from the monotonicity assumption upon $g$ ). Moreover, Fatou's Lemma implies that $t(v)$ maps the space ${W_{0}^{1}}^{12}(\Omega)$ continuously into $R^{1}$ (in the case $N \geq 2$ use the boundedness from below $g$; in the case $N=1$ use the fact that an arbitrary convergent sequence in $w_{0}^{1,2}(\Omega)$ converges uniformly on $\bar{\Omega}$ ).

3. We shall seek a $v \in X$ such that the integral identity 


$$
\begin{aligned}
\sum_{i=1}^{N} \int_{\Omega} \frac{\partial v(x)}{\partial x_{i}} \frac{\partial \psi(x)}{\partial x_{i}} d x-\lambda_{1} \int_{\Omega} & v(x) \psi(x) d x+ \\
& +\int_{\Omega} g\left(t(v) w_{0}(x)+v(x)\right) \psi(x) d x=\int_{\Omega} f(x) \psi(x) d x
\end{aligned}
$$

is valid for an arbitrary $\psi \in X$.

The space $X$ is a closed subspace of $w_{0}^{1,2}(\Omega)$ and hence it is a Hilbert space. It is easy to see that, for a fixed $v \in X$, the mappings

$$
\begin{aligned}
& \mathrm{L}_{v}: \psi \mapsto \sum_{i=1}^{N} \int_{\Omega} \frac{\partial v(x)}{\partial x_{i}} \frac{\partial \psi(x)}{\partial x_{i}} d x-\lambda_{I} \int_{\Omega} v(x) \psi(x) d x, \\
& S_{v}: \psi \mapsto \int_{\Omega} g\left(t(v) w_{0}(x)+v(x)\right) \psi(x) d x-\int_{\Omega} f(x) \psi(x) d x
\end{aligned}
$$

are bounded linear functionals on $X$. Further, according to the Riesz representation theorem there exist uniquely determined elements $L v, S v \in X$ such that

$$
\begin{aligned}
& \langle L v, \psi\rangle=L_{v}(\psi), \\
& \langle S v, \psi\rangle=S_{v}(\psi),
\end{aligned}
$$

for each $v, \psi \in X$.

Let us summarize the properties of the mappings $L$ and $S$.

4. $L$ is a bounded linear operator, and there exists a constant $c>0$ such that

$$
\langle L v, v\rangle \geq c\|v\|^{2}
$$

for each $v \in X$.

5. $S$ is a continuous (generally nonlinear) operator such that, for $v_{1}, v_{2} \in X$, we have

$$
\left(S v_{1}-S v_{2}, v_{1}-v_{2}\right) \geq 0
$$

6. To prove that the boundary value problem (3) is weakly solvable it is sufficient to show that the operator equation

$$
L v+S v=0
$$


has at least one solution in the space $X$.

7. Put $T=L+S$. The mapping $T: X \rightarrow X$ is continuous and strongly monotone, that is to say,

$$
\left\langle T v_{1}-T v_{2}, v_{1}-v_{2}\right\rangle=\left\langle L\left(v_{1}-v_{2}\right), v_{1}-v_{2}\right\rangle+\left\langle S v_{1}-S v_{2}, v_{1}-v_{2}\right\rangle \geq c\left\|v_{1}-v_{2}\right\|^{2}
$$

for all $v_{1}, v_{2} \in X$. Thus, according to the theorem on monotone operators (see, for example, [2]), the mapping $T$ is surjective; that is, $T(X)=X$. Hence the equation (13) is solvable.

\section{References}

[1] A. Ambrosetti, G. Prodi, "On the inversion of some differentiable mappings with singularities between Banach spaces", Ann. Mat. Pura Appl. 93 (1972), 231-246.

[2] Felix E. Browder, Problèmes non linéaires (Séminaire de Mathématiques Supérieures, 15 (Été, 1965). Les Presses de l'Université de Montréal, Montreal, Quebec, 1966).

[3] Svatopluk Fučík, "Surjectivity of operators involving linear noninvertible part and nonlinear compact perturbation", Funkcial. Ekvac. 17 (1974), 73-83.

[4] Svatopluk Fućik, "Nonlinear equations with noninvertible linear part", Caechoslovak Math. J. 24 (99) (1974), 467-495.

[5] Svatopluk Fučlk, "Remarks on a result by A. Ambrosetti and G. Prodi", Bol2. Un. Mat. Ital. (4) 11 (1975), 259-267.

[6] Svatop.luk Fučik, M. Krbec, "Boundary value problems with bounded nonlinearity and general null-space of the linear part", submitted.

[7] Svatopluk Fučík and Milan Kučera and Jindřich Nečas, "Ranges of nonlinear assymptotically linear operators", J. Differential Equations 17 (1975), 375-394.

[8] Jerry L. Kazdan and F.W. Warner, "Remarks on some quasilinear elliptic equations", Comm. Pure Appl. Math. 28 (1975), 567-597. 
[9] E.M. Landesman \& A.C. Lazer, "Nonlinear perturbations of linear elliptic boundary value problems at resonance", J. Math. Mech. $19(1969 / 70), 609-623$.

Department of Mathematical Analysis,

Faculty of Mathematics and Physics,

Charles University,

Prague,

Czechoslovakia. 